\title{
Architecture of dispersed multilayered cyber-physical robotic system for coordination of manipulating robotic system using UAV
}

\author{
Alexey Bulgakov ${ }^{\mathrm{a}}$, Daher Sayfeddine ${ }^{\mathrm{b}}$, Jens Otto ${ }^{\mathrm{c}}$, Sergei Emelianov ${ }^{\mathrm{a}}$ \\ ${ }^{\text {a}}$ Southwest State University,st. 50 let October 94, Kursk, 305049, Russia

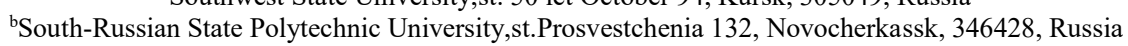 \\ ${ }^{\mathrm{c}}$ Technical University of Dresden, Mommsenstr. 10, Dresden, 01069, Germany
}

\begin{abstract}
Drone technology has demonstrated to be of great contribution to the construction field. Tasks such as photogrammetry, plot surveillance and assets inspections are successfully automated with the aerial robotic technology with some level of contribution of a human operator. In this paper we analyze the possibility to achieve fully automated construction task using dispersed robotic system: a drone and mobile robotic crane. The role of the drone is to study the surroundings and generate a desired trajectory for the crane boom arm in order to lay-in pergola blades. The paper focuses on the layers of the dispersed cyber-physical system allowing to achieve such complex task and optimize the trajectory of the crane boom arm by resolving two-dimensional ChebyshevGauss collocation method in order to have minimum-jerk path.
\end{abstract}

(C) 2019 The Authors. Published by Diamond Congress Ltd.

Peer-review under responsibility of the scientific committee of the Creative Construction Conference 2019.

Keywords: cyber-physical system; drone; trajectory generation; robotics in construction

\section{Introduction}

The practical implementation of the cyber-physical systems in our day to day life is well established. We recognize the development in such solutions leading to Internet of Things (IoT), augmented and virtual reality contributing to a new level of end-user's satisfactions and extended useful experience. In contrast, the paradigm of applying cyberphysical systems to the construction field is yet to explore and set its own potential. This is majorly due to the late and slow development of the autonomous and robotized platforms which has a projected application in the construction field.

A techno-commercial solution of the autonomous systems in construction is rarely considered as it is always cheaper and less risky to deploy man-power (carpenters, steel fixers) rather than purchasing and customizing a robotic solution. In one hand, the implementation of sophisticated solution is costly and require specialized staff present at site for monitoring, and on the other hand, the solution is not widely tested and its results are barely registered and recognized. 
Alexey Bulgakov, et al./ Proceedings of the Creative Construction Conference (2019) 003

https://doi.org/10.3311/CCC2019-003

In this paper, we tackle the first challenge aiming to reduce the specialized and idle man power by studying the automation level required to lay-in pergola blades on top of a building using dispersed cyber-physical system composed of a drone and a mobile lifting crane. By cyber-physical system, we understand the concept of reliable fusion between computational and physical platform embedded within the same body [1], however, enabling better interaction capabilities with the surrounding (world and human operator) though many modalities and processes. In light of that, the terms of interaction, integration and enhancement are key areas where the research of cyber physical systems is occurring.

Traditionally, the physical system is a consolidated system and not dispersed over selected area. Recently, with the introduction of Internet of things, the probability of designing distributed physical systems became achievable, as it overcame the disadvantages of radio systems and the huge cost related to GPS and GLONASS integration in navigation platforms, which made this solution not optimal techno-commercially. In this paper, we introduce the cyber-physical system consisting of the following levels:

- Aerial: a UAV scanning the roof and generating the positioning tasks for the subsequent component of the pergola;

- Cybernetic: analyzing the video-input of the UAV and generating trajectory for the manipulator using visual odometry algorithm;

- Ground: a Mobile robotic crane, receiving the coordinates from the cyber system and performing the layingout of the pergola blades.

A concept of the solution is illustrated in fig. 1 according to which, the UAV will dictate the following parameters as a potential control task to the cybernetic system: the swing angle $\phi$, the luff angle $\theta$, the hoist elevation $h$ and the mobility across axis $\mathrm{OX}$ and $\mathrm{OY}$, which can be replaced by the angle formed by the hoist and the luff axis [2].

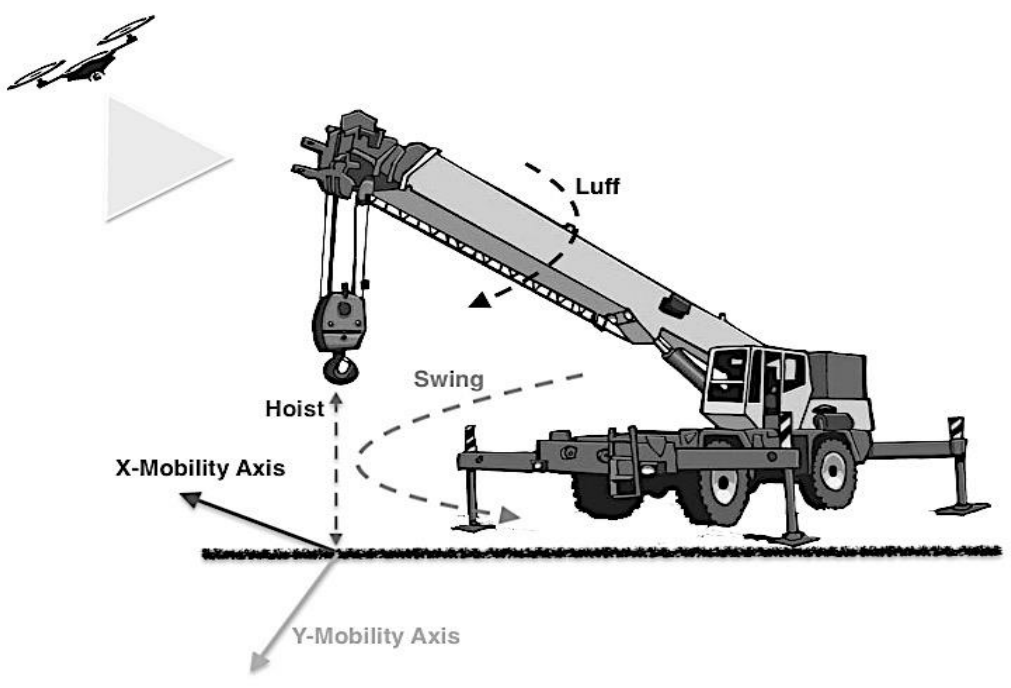

Fig 1. Concept of dispersed cyber-physical system

\section{Paradigm of Proposed Cyber Physical System}

By paradigm, we underline a worldview underlying theories and methodologies of a particular scientific subject. To some extent, the notion of the cyber-physical system is well defined in the IT fields. However, its application in other engineering subjects is yet to be defined. Our target in this paper is to make notions more tangible and hence allowing better evaluation and optimization. In line of that, we will be studying the generic system architecture, the enabling knowledge and technology assets, and distinguishing system characteristics allowing for expansion and optimization. 
Alexey Bulgakov, et al./ Proceedings of the Creative Construction Conference (2019) 003

https://doi.org/10.3311/CCC2019-003

Cyber physical systems (CPS) have emerged from the synergetic merging of information, sensory and communication system with a physical system. This is a comparable notion to mechatronic system, from where we will begin integrating CPS architecture to the electromechanical word of construction. In addition, we have to consider the human-machine interface as the CPS will not be deployed at site in isolation with the other construction activities. Hence, an additional layer in the CPS will be figuring out and influencing on the overall performance of the system. Fig.2 illustrate generalized CPS layers and compared to the applied proposed CPS [6,7].
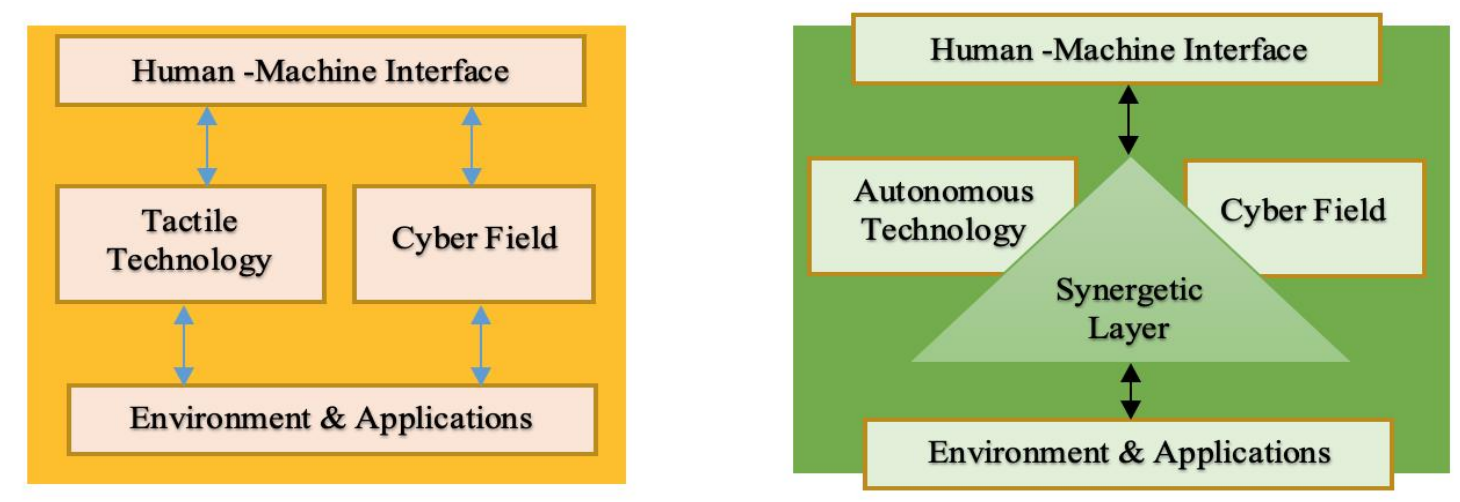

Fig 2. Traditional and Proposed CPS

As it can be seen from fig.2, the difference between a traditional and the proposed CPS can be highlighted in several points: firstly, the human-machine interface is encompassed inside the CPS in the traditional system, however, in the proposed layout, the layer is peripheral and has no direct interaction with the autonomous technology and cyber field. Secondly, the tactile technology is substituted with an autonomous system as we are aiming to robotize construction task and lastly, the direct interaction with the environment and application is done by a synergetic "mechatronic layer" responsible of communication and execution of the task. In addition, the environment layer is neither connected directly to the autonomous and cyber system. In short, the difference lays in omitting the human factor influencing the process and replacing it with actuating mechanism.

The proposed model complies with the worldwide notions of CPS especially point C5 stating the system should be articulated and heterogenous. By articulated, we define the connectivity of the system with its embedded components and environment; and by heterogenous- we mean the different physical origin of each systems: having a cyber part, a synergy and actuators. The same is import as the CPS can suffer possible downtime leading to task failure. In this case, the CPS analysed by its Cyber part has to plan for a functional (graceful) degradation [3].

\section{System Architecture}

In this paragraph, we will discuss the core parts of the CPS, its parameters, requirement and functions. We will study the cyber system component, set the algorithm of trajectory generation for the crane boom arms using UAV and identify the transformation equations between the pixel and metric references. The last part of the paragraph will address the optimization of the generated trajectory and transformation of the boom arm movement to a twodimensional Chebyshev-Gauss collocation method in order to achieve minimum jerk movement considering zero initial conditions.

\subsection{Cyber system}

Firstly, regarding the cyber field, an embedded software command the generation of the trajectory of the crane boom arms and requirement of the geometric coordinates explained in paragraph1. In addition, the cyber system is responsible of intra and inter-objects transmissions. As per the current standards of CPS the following range of transmission for a multi-scale CPS need to be identified: 
Alexey Bulgakov, et al./ Proceedings of the Creative Construction Conference (2019) 003

https://doi.org/10.3311/CCC2019-003

\begin{tabular}{llll}
\hline \multicolumn{3}{c}{ Tab.1 Transmission Range for Intra and Inter-Objects communication } \\
\hline \multicolumn{2}{l}{ Intra-Object Transmission } & Inter -Objects Transmission \\
\hline \multirow{2}{*}{ Not Available } & Global & $10^{6} \mathrm{~m}$ \\
Nano Scale & from $10^{-9} \mathrm{~m}$ & Long range & $10^{4} \mathrm{~m}$ \\
Micro Scale & Up to $10^{-6} \mathrm{~m}$ & Medium Range & $10^{2} \mathrm{~m}$ \\
Macro Scale & Up to $10^{-3} \mathrm{~m}$ & Short Range & $10^{0} \mathrm{~m}$ \\
\hline
\end{tabular}

From tab.1, we need to pay attention to the following: the intra-object transmission needs to be respected and achieved by all means, since it is a mandatory requirement for possible functional degradation in case of system failure. Pertaining to the inter-objects' transmission, a least of medium range should be achieved allowing the communication between the drone and the crane with possible engagement from the human-machine interface.

The other part of the cyber system is the trajectory generating software. We will be deploying the drone, equipped with a camera, to generate desired trajectory for the boom arm, study possible collision and assign subsequent step to lay the pergola blade. Using machine vision and odometry, CPS can autonomously define the trajectory of the boom arm. Figure 3 and equations (1-3) illustrate the transition between pixel to metric coordinates.

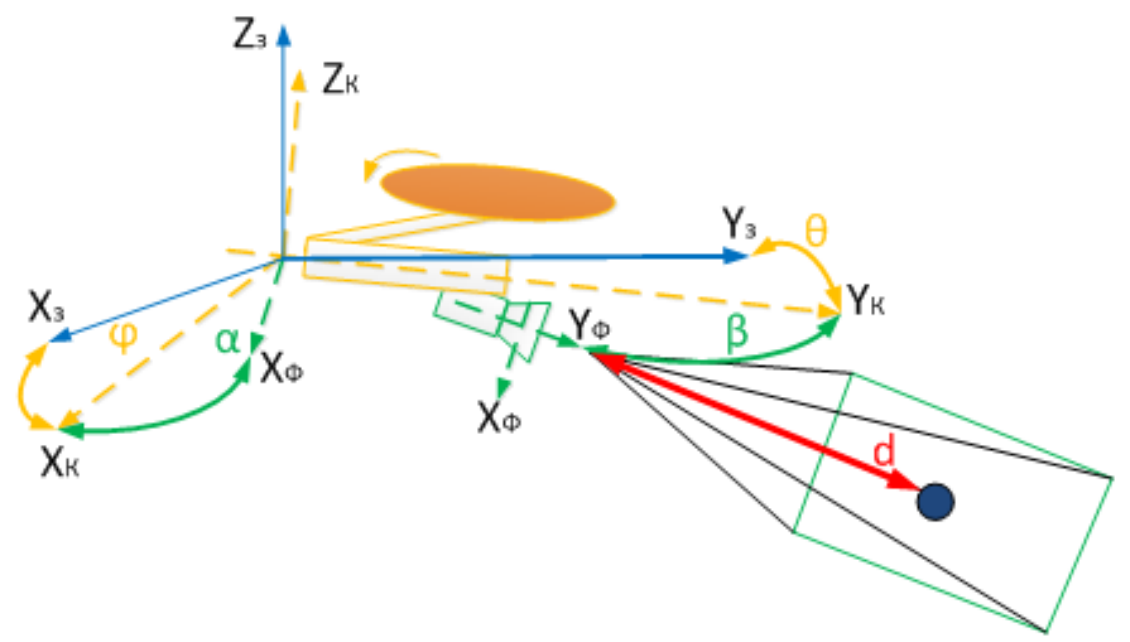

Fig 3. Illustration of different reference systems and coordinates

The distance from the camera to a specific coordinate (Earth-axis) is computed using the following equations:

$$
\begin{aligned}
& d(x)=H_{\Gamma}=H * \tan (\varphi+\alpha) ; \\
& d(y)=H_{\mathrm{B}}=H * \tan (\theta+\beta),
\end{aligned}
$$

Using equations (7) and (8), we can identify the transformation formulas between different reference systems.

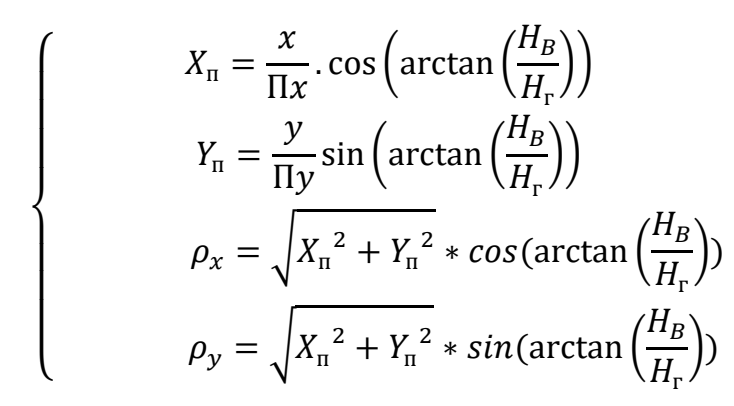


Alexey Bulgakov, et al./ Proceedings of the Creative Construction Conference (2019) 003

https://doi.org/10.3311/CCC2019-003

Where $X_{п}$ and $Y_{п}$ - are the pixel coordinates, $x$ and $y$ - are the body axis coordinate and $\rho_{x}$ and $\rho_{y}$ are the angular pixel coordinates.

\subsection{Actuating System}

The actuators are responsible of fulfilling the generated trajectory, to lift and lay the pergola blades according to the defined coordinates. In addition, the process has to be jerk-free movement in order to avoid collisions and inverted pendulum effect, especially that wind might affect the positioning of the blade at a certain height. To achieve an optimal trajectory to the manipulator, the movement will be posed and solved as quadratic programming using multi-segment Chebyshev orthogonal collocation for transcription. Using the two-dimensional Chebyshev-Gauss collocation method to obtain possible numerical solution of differential equation by partially is differentiating it in time and discretizing it using finite difference method [4]. Taking into consideration that the trial or candidate trajectories $f_{k}(x)$ will be set on homogenous Dirichlet boundary conditions [3], the approximate solution can be presented in the following equation

$$
U^{N}(x, t)=\sum_{k=0}^{N} a_{k}(t) f_{k}(x)
$$

In the collocation approach, the differential equation has to be satisfied by the approximate solution at the collocation points in the assigned domain [5]. The derivative at each node can be found by multiplying the value at any Chebyshev point by a differentiation matrix.

\section{Results}

By implementing the proposed model, and running the quadratic programming listing on Matlab, we obtained the result of multi-segment trajectory simulation as depicted in figure 4.
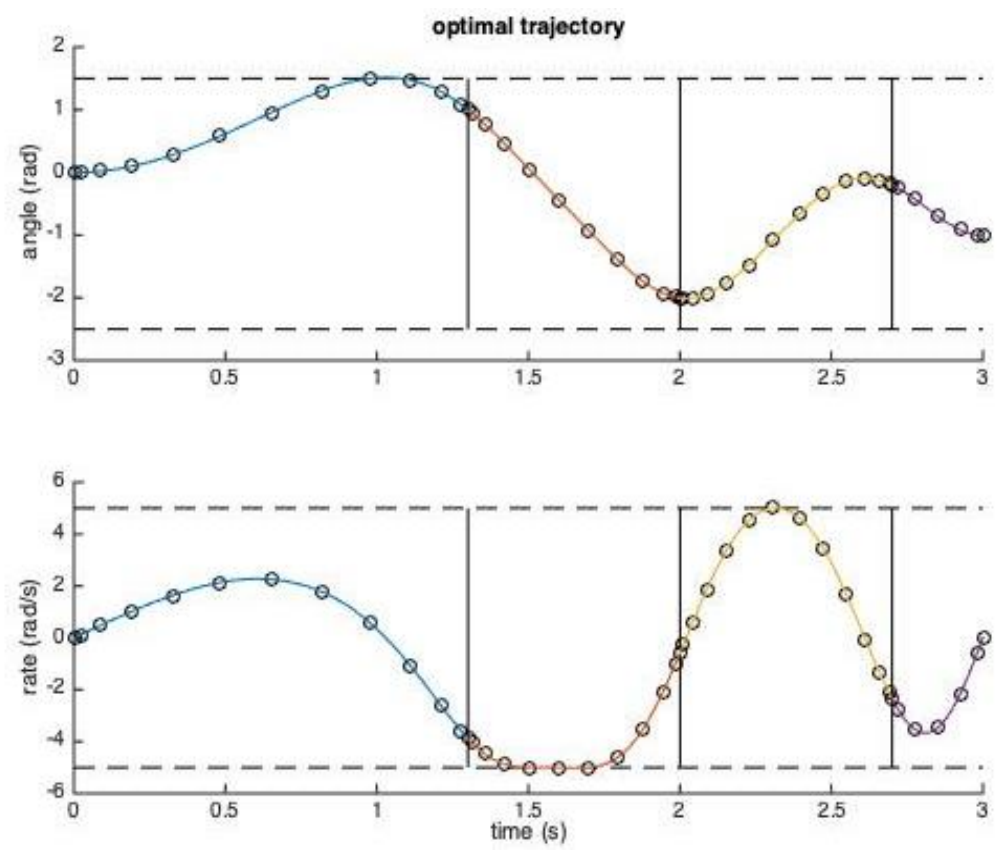

Fig. 4. Results of multi-segment trajectory

In figure 4, we can clearly see, that the trajectory is passing by all the Chebyshev node points, where the initial a final velocity rated in $\mathrm{rad} / \mathrm{s}$ is zero and the angle of the final trajectory is almost negligible.

By applying the same concept to the both sections of the manipulator, with specific boundaries and input trajectory, we can see that the arm end-effector holding the bald is travelling smoothly, on the doted lines, shown in fig.5, taking 
Alexey Bulgakov, et al./ Proceedings of the Creative Construction Conference (2019) 003

https://doi.org/10.3311/CCC2019-003

into consideration for joint 1 and 2, the initial velocity is zero (starting point of lifting task), and the resultant final velocity of the end-effector is also zero rad/s, which corresponds to discharge of the manipulator after laying task.

\section{Conclusion}

This paper studied the architecture of multilayered cyber-physical system consisting of UAV and robotic crane. The role of the UAV is to generate desired path for the crane boom arm in order to lift and lay pergola blades on top of a building. The paper addressed different layers of the cyber physical systems and proposed an additional synergetic part interacting solely with human machine interface and aquire information for the environment. In such scenario the cyber physical system will be bordered and allow for better functional (graceful) degradation if required. Later, the paper focused on the trajectory planning and generation of transportation coordinates of the pergola blades. The problem is
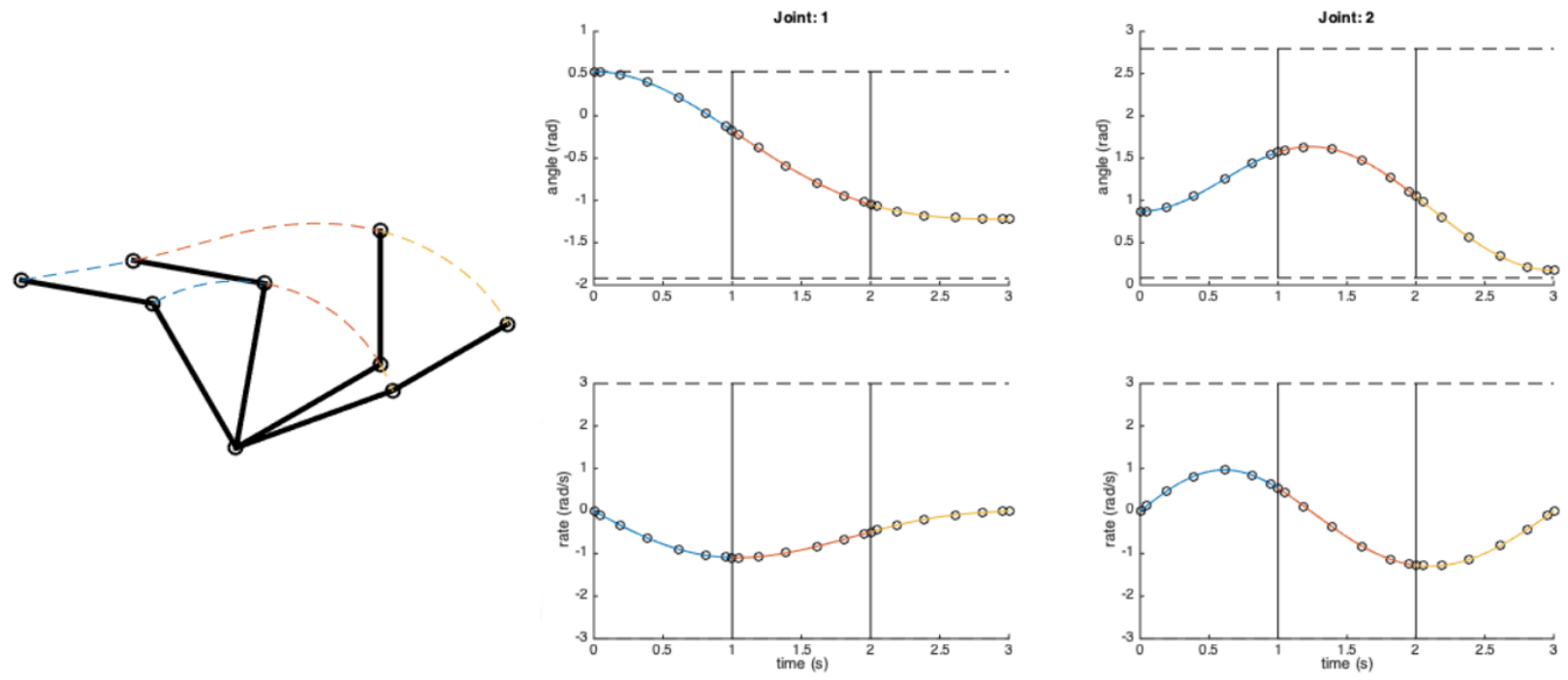

Fig 5. Minimum-Jerk Trajectory of the robotic manipulator corresponding to Chebyshev nodes

then transformed as two-dimensional Chebyshev-Gauss orthogonal collocation method aiming to achieve jerk-free transportation and positioning of the blades. The obtained simulation results illustrated both the joints of the lifting crane have followed the optimal trajectory, passing by all Chebyshev points with minimum jerk considering that the initial velocity of the joints is zero and the final velocity should be obtained zero rad/s with minimum to zero overshooting angles.

\section{References}

[1] A. Rajhans et al. An architectural approach to the design and analysis of cyber physical systems. 3rd International workshop on multiparadigm modelling

[2] A. Upadhay et Al. UAV-Robot relationship for coordination of robots on a collision free path. International conference on robotics and smart manufacturing (RoSMa2018), pp. 424-431 https://doi.org/10.1016/j.procs.2018.07.052

[3] Cheng, A. and D. T. Cheng. Heritage and early history of the boundary element method, Engineering Analysis with Boundary Elements, 29, 268-302 https://doi.org/10.1016/j.enganabound.2004.12.001

[4] M. Sameeh et Al. Chebyshev Collocation Method for Parabolic Partial Integrodifferential Equations. Advances in Mathematical Physics Volume 2016, Article ID 7854806, 7 pages http://dx.doi.org/10.1155/2016/7854806

[5] L. Yixin. Introduction to spectral methods

[6] I. Horvath, B Gerritsen. Cyber-physical systems: concepts, technologies and implementation principles. Proceedings of TMCE 2012, May 711, 2012, pp. 19-36

[7] Rajhans, A., Cheng, S.-W., Schmerl, B., Garlan, D., Krogh, B. H., Agbi, C. and Bhave, A., 2009, “An architectural approach to the design and analysis of cyber-physical systems", in Proceedings of the 3rd International Workshop on Multi-Paradigm Modeling - MPM 2009, EASST, Vol. 21, pp. 1-10. https://doi.org/10.1016/j.procir.2016.11.007

[8] D. Sayfeddine. Functional degradation of cyber-physical aerial system for trajectory planning and agent tracking. The International Conference on Information Technologies in Business and Industry, 2019. 6 pages. 